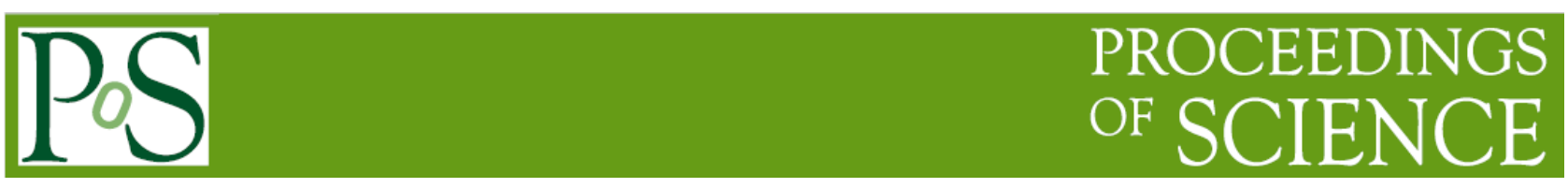

\title{
Some Ideas and Designs for Simplification and Modification of Cavity-based Dark Matter Searches
}

\author{
Masroor H. S. Bukhari \\ Department of Physics, Jazan University, \\ Gizan, Jazan 45142, Saudi Arabia \\ E-mail: mbukhariejazanu.edu.sa
}

A proposal comprising some ideas and designs is reported here for a simplified signal detection scheme to aid in cavity-based Dark Matter searches, especially for Skivie axions. An enhanced and more sensitive on-resonance signal detection scheme is proposed incorporating a modified Colpitts oscillator and simple read-out electronics which simplifies the electronics associated with usual DM search experiments. In addition, we suggest the use of Halbach geometry magnets to eliminate the need for expensive and large electromagnets, which pose a number of problems including background noise and arduous handling. We hope that these ideas could be useful in improving and simplifying cavity-based cold dark matter searches.

38th International Conference on High Energy Physics

3-10 August 2016

Chicago, USA

(c) Copyright owned by the author(s) under the terms of the Creative Commons

Attribution-NonCommercial-NoDerivatives 4.0 International License (CC BY-NC-ND 4.0). 


\section{- Introduction}

Among various ways to detect light cold Dark Matter (DM) particles, such as axions, present in the DM halo of our galaxy, a technique has been proposed for these to be resonantly detected in a microwave cavity in a strong transverse magnetic field, under an effect known as the Inverse Primakoff effect [1]. It was proposed in Eighties [2] that with the help of a strong magnetic field, an effective axion-photon conversion can take place, with the generation of a microwave photon within the resonant cavity.

In this regard, there are a number of experiments and searches [3,4], based on the implementation of the Inverse Primakoff effect to search for these axions (generally known as Skivie Axions), spanning a reasonably vast mass range. These experiments are formed around a high-quality factor resonant cavity, which is tuned with a tuning rod across the range of frequencies of interest, and the possible signals are picked up by an antenna and amplification scheme built around a SQUID (Superconducting Quantum Interference Device) and an HFET (Heterogeneous Field Effect Transistor) device [3], and associated RF electronics. The detector assembly is housed in a cryostat, operating at sub-Kelvin temperatures.

\section{- Our Proposed Scheme}

We propose a detection scheme by presenting a resonance detection methodology which might aid in measuring onresonance signals from possible axion-photon conversion events, while offering possible improvements in all these areas. A new and innovative detection element (the Tunnel Diode-HEMT duo) is designed and proposed to work in conjunction with the SQUID to aid in resonance detection, a simpler $d c$ resonance detection and measurement method replaces the complicated RF detection scheme, and we suggest a halbach permanent magnet array to offer an alternative to the electromagnet option (however, this is just a minor improvement, the scheme could still work with the conventional methods as employed in on-going searches).

Our hypothesis is based on a tenet that axions could follow a specific mass distribution function and thus a given flux of axions could be populated by a distribution of axion masses. To begin with, we probe an axion mass $\left(\mathrm{m}_{\mathrm{a}}\right)$ range of approximately $8 \times 10^{-6}$ to $12.5 \times 10^{-6} \mathrm{eV}$ (which corresponds to a narrow frequency spectrum $\left(f_{a}\right)$ corresponding to $2.0 \mathrm{GHz}$ to $2.99 \mathrm{GHz}$ ), for which we have developed appropriate cavities and associated instrumentation, as summarized in this preliminary strategy report.

Our scheme, a simple form of which can be depicted with the help of a diagram in Figure 1, revolves around enhancements in resonance detection methodology and simplifications in the signal read-out by employing a $d c$ read-out scheme. In addition, we propose the use of permanent halbach magnets to facilitate the required magnetic field. In this connection, we present the idea of using a tunnel diode (TD) [5] installed at the cavity along with the SQUID serving as the main detector, coupled to a cryogenic Ultra-Low Noise Amplifier (ULNA), which is nothing but a modified low-temperature and very-low noise form of a conventional Low-Noise Amplifier (LNA) [6]. The Tunnel Diode and HEMT amplifier in conjunction with the cavity LC tank induce resonance resonance with the electromagnetic fields present in the cavity, enhancing the power of possible weak photons produced as a result of axion conversions.

We also propose the use of axial halbach geometry [7] permanent magnet arrays to reduce the electromagnetic interference and other problems in the experiment. In these magnet arrays, the magnetic field is concentrated inside the bore of the magnet with vanishing fields outside.

More details of our proposal and some preliminary test results, as well as designs of our instrumentation (especially the ULNA's and detectors) are discussed in detail in $[8,9]$. 


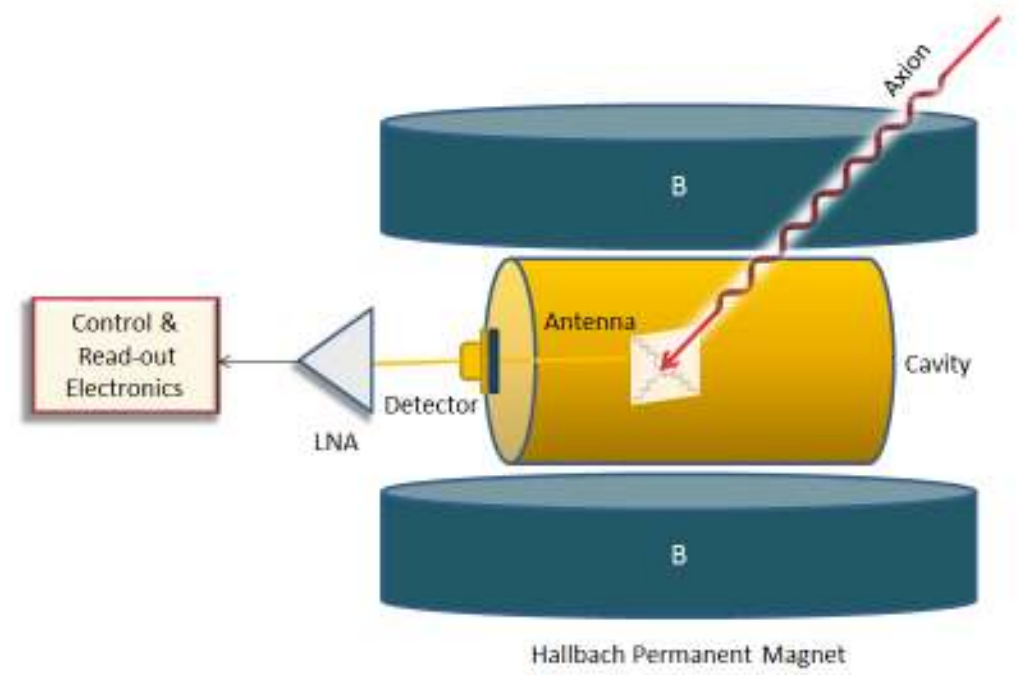

Figure 1. Our proposed experiment and detection scheme.

Although our proposed scheme has not been implemented in an actual axion detection experiment so far, we present some results of artificial axion signals 'injected' into a model cavity to evaluate the scheme's suitability. An axion 'event' of mass range $10-11 \mu \mathrm{eV}$ was simulated with the help of a $2.4-2.5 \mathrm{GHz}$ signal and weakly coupled to the cavity, and its resonance was detected, by measuring the voltage changes with the help of our proposed detectoramplifier scheme. Figure 2 illustrates a plot of measured raw voltage for the aforementioned test signal weakly coupled to the cavity.

Our initial test results illustrate that our ideas and the proposed scheme constitute good potential for use in serious cold dark matter searches.

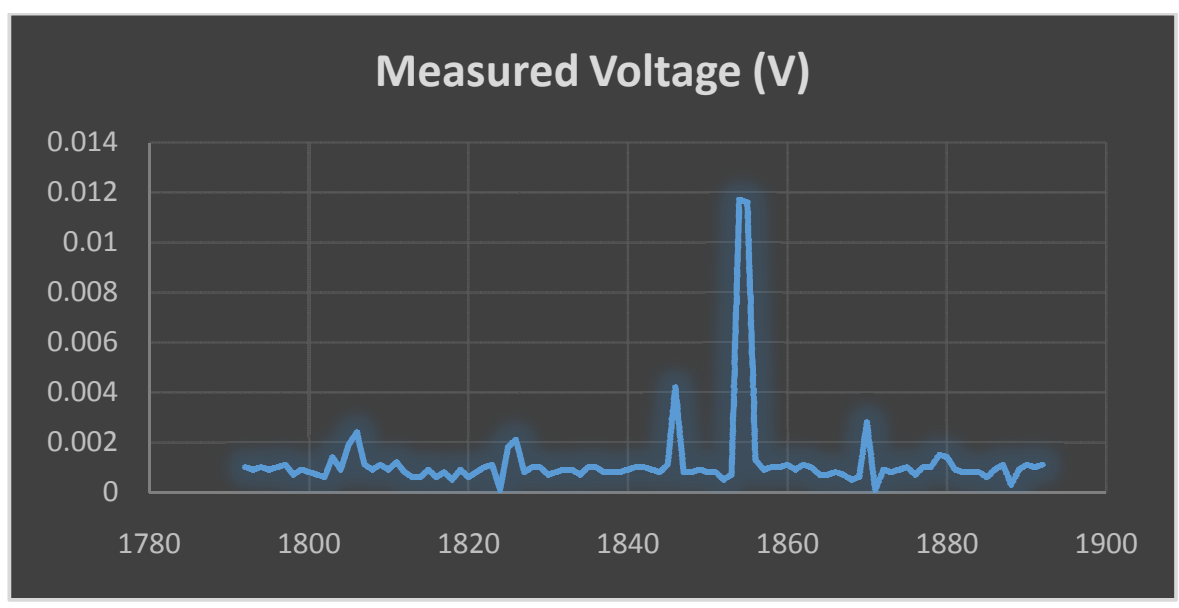

Figure 4: An artificial axion signal injected into cavity measured by our scheme.

Funding Support: Funding support from the King Abdul Aziz City of Science and Technology (KACST), Saudi Arabia, under the KACST Small Grant program (Grant number 234-35), is acknowledged with gratitude. 


\section{References}

[1] D.A. Dicus et al., Astrophysical bounds on the masses of axions and Higgs particles, Phys. Rev. D18 (1978) 1829.

[2] P. Sikivie, Experimental Tests of the Invisible Axion, Phys. Rev. Lett. 51 (1983) 1415 [Erratum ibid. 52 (1984) 695].

[3] S. J. Asztalos et al., SQUID-Based Microwave Cavity Search for Dark-Matter Axions, Phys. Rev. Lett. 104 (2010)041301.

[4] L. D. Duffy et al.,. High resolution search for dark-matter axions, Phys. Rev. D74 (2006) 12006.

[5] Electronics Engineers Reference Book, L. V. Turner (Ed.), $4^{\text {th }}$ Ed., Butterworth-Heinemann, London, 1976, pp. $8-18$ to $8-19$

[6] P. Horowitz and W. Hill, The Art of Electronics, $2^{\text {nd }}$ Edition, Cambridge, 1989.

[7] K. Halbach, Design of permanent multipole magnets with oriented rare earth cobalt material, Nuclear Instruments and Methods (1980) 169, 1-10; ibid.NIM 187 (1981) 109

[8] M. H. S. Bukhari and Z. H. Shah, A Detection Scheme for Cavity-based Dark Matter Searches, arXiv:1604.06900v2 [physics.ins-det]

[9] M. H. S. Bukhari and Z. H. Shah, Low-Noise Amplification, Detection and Spectroscopy of Ultra-Cold Systems in RF Cavities, Modern Instrumentation 5 (2016) 5-16. doi: 10.4236/mi.2016.52002. 